\title{
MR Features Based on LI-RADS Ver. 2018 Correlated with Cytokeratin 19 Expression in Combined Hepatocellular Carcinoma-Cholangiocarcinoma
}

\author{
Changwu Zhou ${ }^{1,2, *}$ \\ Xiaoyan $\mathrm{Ni}^{2}$,* \\ Xin $\mathrm{Lu}^{2}$ \\ Yi Wang ${ }^{2}{ }^{2}$ \\ Xianling Qian ${ }^{2}$ \\ Chun Yang ${ }^{2}$ \\ Mengsu Zeng ${ }^{1,2}$
}

'Shanghai Institute of Medical Imaging, Shanghai, People's Republic of China; ${ }^{2}$ Department of Radiology, Zhongshan Hospital, Fudan University, Shanghai, People's Republic of China

*These authors contributed equally to this work
Correspondence: Chun Yang Department of Radiology, Zhongshan Hospital, Fudan University, Shanghai, People's Republic of China

Tel +86 I8702I35336

Email dryangchun@hotmail.com

Mengsu Zeng

Shanghai Institute of Medical Imaging,

Shanghai, People's Republic of China

Tel +8613501922963

Email zeng.mengsu@zs-hospital.sh.cn
Purpose: To investigate the significance of MR features based on the Liver Imaging Reporting and Data System (LI-RADS ver. 2018) for identifying the expression of cytokeratin 19 (CK-19) in patients with combined hepatocellular carcinoma-cholangiocarcinoma (cHCC-CCA) before surgery.

Patients and Methods: The study enrolled 174 patients pathologically confirmed to have cHCC-CCA according to the 2019 WHO classification. The preoperative MR imaging features and clinicopathological findings were retrospectively evaluated and compared between the CK-19-positive and CK-19-negative cHCC-CCA groups.

Results: One hundred seventy-four patients (mean age, males vs females: $56.6 \pm 10.0$ years vs $54.7 \pm 14.2$ years) were evaluated. The presence of mosaic architecture, targetoid appearance, cholangiectasis, hepatic capsule retraction, and corona enhancement was significantly higher in the CK-19-positive group (all $\mathrm{p}<0.05$ ), while nonrim arterial phase hyperenhancement (APHE) was more common in the CK-19-negative group $(p=0.04)$. The univariate analysis showed that hepatitis $\mathrm{B}$ virus infection, CEA $>5 \mathrm{ng} / \mathrm{mL}$, tumor size, nonrim APHE, mosaic architecture, targetoid appearance, cholangiectasis, hepatic capsule retraction, and corona enhancement were significant risk factors for CK-19-positive cHCCCCA (all $\mathrm{p}<0.05$ ). Unfortunately, the multivariate analysis revealed that only corona enhancement $(\mathrm{OR}=2.359, \mathrm{p}=0.03)$ was an independent risk factor associated with $\mathrm{CK}-19$ positive cHCC-CCA.

Conclusion: Corona enhancement is significantly correlated with CK-19 positivity in patients with cHCC-CCA.

Keywords: liver neoplasms, magnetic resonance imaging, cytokeratin 19

\section{Introduction}

Combined hepatocellular carcinoma-cholangiocarcinoma (cHCC-CCA) requires more attention due to its heterogeneous characteristics, biological behavior, and dilemmas in its diagnosis. ${ }^{1,2}$ Misdiagnosis without histopathological confirmation can result in improper treatments for cHCC-CCA. ${ }^{3}$ All these factors may be challenging in treatment decision-making and in evaluating the prognosis of $\mathrm{cHCC}$ CCA patients.

Cytokeratin 19 (CK-19) is currently considered as a marker for the biliary type and is expressed in hepatocellular carcinoma (HCC) and intrahepatic 
cholangiocarcinoma (ICC). ${ }^{4-6}$ In previous studies, some findings have suggested that HCC with CK-19 expression is significantly correlated with adverse clinicopathological outcomes, such as tumor aggressiveness, vascular invasion, poor disease-free survival and overall survival. ${ }^{7-9}$ Similarly, CK-19 is highly expressed in cHCC- CCA (2019 WHO classification) displaying both hepatocellular and cholangiocellular differentiation. cHCC-CCA with CK-19 expression also tended to show poorer overallsurvival and disease-free survival. ${ }^{6}$ Therefore, the status of CK-19 in cHCC-CCA was as important as its morphological classification.

cHCC-CCA lesions can be categorized based on the Liver Imaging Reporting and Data System (LI-RADS ver. 2018) using major features to mimic HCC, including nonrim arterial phase hyperenhancement (APHE), nonperipheral washout, capsular enhancement, and targetoid appearance. $^{10,11}$ Studies have indicated that some MR imaging features, such as arterial phase irregular rim enhancement, hypovascularity in the arterial phase, nonperipheral washout, and targetoid appearance, are significant independent predictors of CK-19-positive HCC. ${ }^{12-14}$ Furthermore, we should explore the usefulness of MR imaging features for identifying CK-19 expression in cHCC-CCA lesions. Therefore, the purpose of this study was to determine whether major MR features based on LIRADS ver. 2018 can aid in investigating the expression of CK-19 in patients with cHCC-CCA before surgery.

\section{Patients and Methods}

\section{Patients Selection}

This retrospective study was approved by the Institutional Review Board of Zhongshan Hospital, Fudan University (Approval No.: B2021-325R), and a written consent form was required from each patient before enrollment. In total, 204 consecutive patients with cHCC-CCA were first identified by reviewing postoperative pathology between June 2016 and March 2021. One hundred seventy-eight patients were included in accordance with the following criteria: (1) pathologically confirmed cHCC-CCA according to the updated 2019 WHO classification system ${ }^{15}$ after surgery (all the pathology results were reanalyzed and the category of cHCC-CCA with stem cell features was excluded); (2) preoperative contrast enhancement MR imaging; and (3) the availability of an immunochemical marker of CK-19. Some patients were excluded due to (1) insufficient quality for MR images $(\mathrm{n}=1)$; (2) curative treatment before MR imaging $(\mathrm{n}=2)$; and (3) incomplete clinical data of tumor biomarkers $(\mathrm{n}=1)$. Finally, 174 cHCC-CCA patients were enrolled in this study. The flowchart of patient enrollment is displayed in Figure 1.

This study had some overlap with a published study ${ }^{16}$ and a recently accepted study in terms of patients. In the published study, 113 patients were included to predict microvascular invasion by conventional MRI findings. In the recently accepted study, $160 \mathrm{cHCC}-\mathrm{CCA}$ patients were enrolled to investigate the prognostic factors for recurrence-free survival and overall survival according to the imaging characteristics. However, the current study enrolled $174 \mathrm{cHCC}-\mathrm{CCA}$ patients and investigated the predictive factors for $\mathrm{CK}-19$ in relation to the clinicopathological and MR imaging characteristics.

\section{Clinical and Pathological Data Evaluation}

All clinical information of the cHCC-CCA patients, including age, sex, hepatitis $\mathrm{B}$ virus infection status, and levels of tumor markers such as serum AFP, CEA, and CA19-9 within 7 days before curative resection, was retrospectively collected from the medical records. The cutoff values for AFP, CEA, and CA19-9 were $20 \mathrm{ng} / \mathrm{mL}, 5 \mathrm{ng} /$ $\mathrm{mL}$, and $37 \mathrm{U} / \mathrm{mL}$, respectively. The pathological findings of the lesions included CK-19, MVI, and tumor size $(\leq$ $2 \mathrm{~cm}, 2-5 \mathrm{~cm}, \geq 5 \mathrm{~cm}$ ).

In this study, all patients were divided into a CK-19positive group and a CK-19-negative group according to their CK-19 levels. CK-19 positivity was defined as membranous and/or cytoplasmic expression in $\geq 5 \%$ of tumor cells with moderate or strong intensity. Then, the differences in clinical and pathological data were compared between the CK-19-positive group and the CK-19negative group.

\section{MRI}

All patients were performed with a 24-channel 1.5 T MR scanner (uMR 560, United Imaging Healthcare). Precontrast liver protocols consisted of transverse T2 weighted imaging (T2WI), in-phase and opposed-phase sequences, T1WI, and diffusion-weighted imaging (DWI, $\mathrm{b}$ value $=0,50$, and $500 \mathrm{~s} / \mathrm{mm} 2$ ). Dynamic contrastenhanced MR imaging was operated with a T1-weighted fat-suppressed sequence. After intravenous administration at a dose of $0.1 \mathrm{mmol} / \mathrm{kg}$ at a rate of $2 \mathrm{~mL} / \mathrm{s}$, the arterial phase was acquired when the contrast agent (gadolinium diethylenetriamine pentaacetic acid, Gd-DTPA; Magnevist, Bayer HealthCare) reached the ascending 


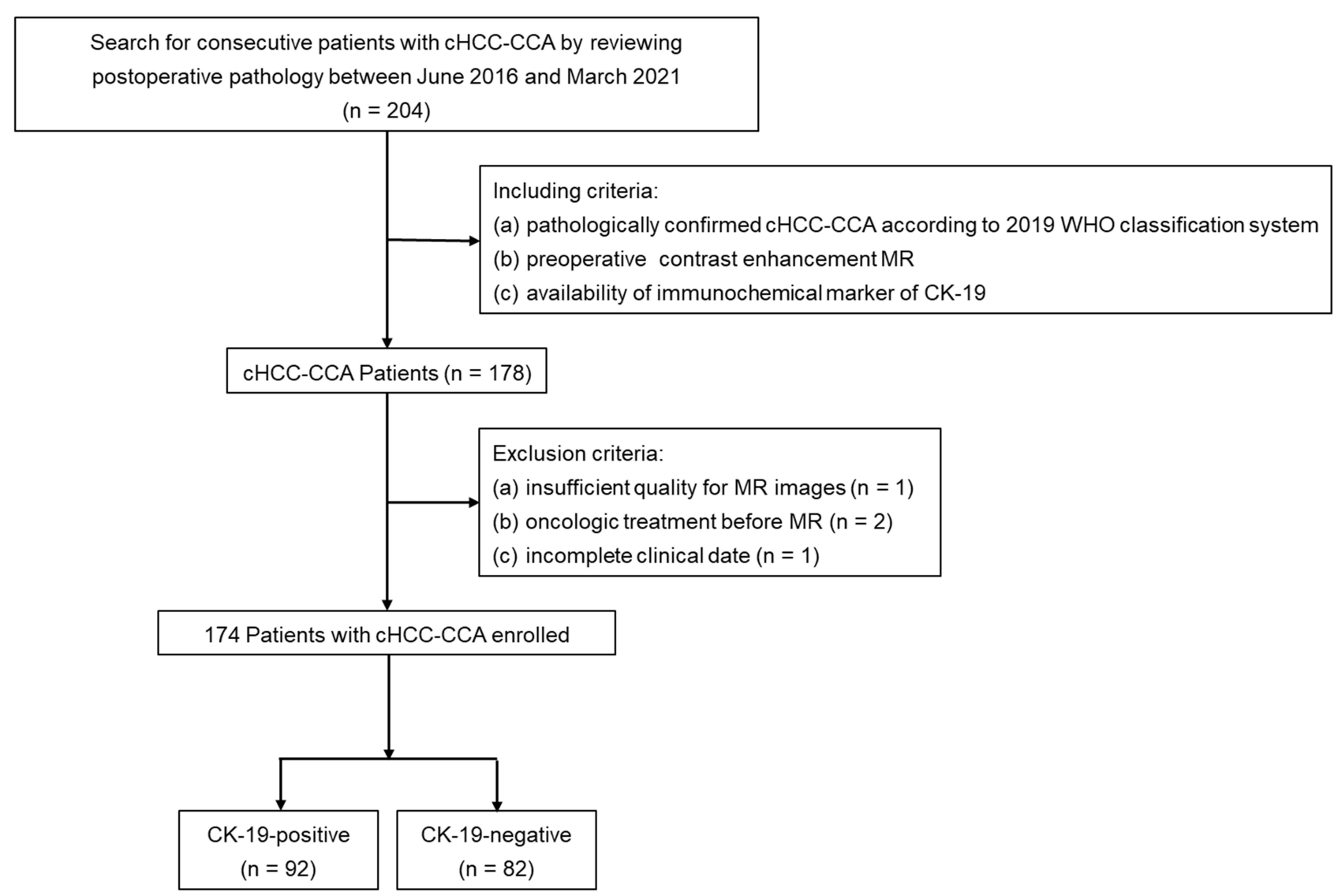

Figure I Flowchart of this study cohort.

Abbreviations: CHCC-CCA, combined hepatocellular carcinoma-cholangiocarcinoma; CK-19, cytokeratin 19.

aorta. Then the portal venous phase and delayed phase sequences were acquired at 70-90 $\mathrm{s}$ and $160-180 \mathrm{~s}$, respectively. All the sequences with the detailed parameters are found in a published paper. ${ }^{16}$

\section{Image Features Interpretation}

Two radiologists (C.W.Z. and C.Y., who have 12 and 14 years of experience in abdominal imaging, respectively) retrospectively investigated all MR images. They were blinded to the clinical data, tumor markers, and pathological results but were aware that the patients had cHCCCCA. They evaluated MR images independently. A consensus was negotiated when there was disagreement between the two observers.

The following imaging characteristics of cHCC-CCA were investigated on precontrast MR images: (a) restricted diffusion, (b) intratumoral hemorrhage, (c) cholangiectasis (peritumoral bile duct dilatation), and (d) hepatic capsule retraction. In addition, the following dynamic enhancement features based on LI-RADS ver. 2018 were evaluated: (A) arterial phase: (a) nonrim arterial phase hyperenhancement
(APHE) and (b) corona enhancement (presence or absence, defined as the hyperperfusion of liver tissue surrounding the tumor border); (B) portal venous phase: (c) nonperipheral washout, (d) enhancing capsule (presence or absence), and (e) portal vein thrombus (presence or absence); (C) delayed phase: (f) delayed central enhancement (presence or absence); and (D) other imaging features: (g) nodule-innodule architecture (presence or absence) and (h) mosaic architecture (presence or absence). In addition, targetoid appearance (target sign on DWI and rim APHE, peripheral washout, and delayed central enhancement on dynamic contrast-enhanced MR imaging), as a criterion for LR-M, and LI-RADS categorization was also assessed in this study. Comparisons of imaging features were performed between the CK-19-positive and CK-19-negative cHCC-CCA groups.

\section{Statistical Analysis}

Statistical analyses were performed by using SPSS 26.0 (IBM). Data with a normal distribution are presented as the mean \pm standard deviation, and the differences between the two groups were compared by using an 
independent-sample $t$-test. Moreover, categorical variables are shown as the number of cases and the percentages, and comparisons between these groups were performed by using the chi-square test or Fisher's exact test. Furthermore, univariable logistic regression analysis was performed to identify which of the patients' clinicopathological features and MR imaging characteristics could be helpful to discriminate between the CK-19-positive group and the CK-19-negative group as the dependent outcome. Subsequently, variables with $\mathrm{P}<0.05$ in univariate analysis were entered into multiple logistic regression analysis to identify significant independent risk factors with the odds ratio (OR) and 95\% confidence interval (CI). Differences with a $\mathrm{p}$ value of $<0.05$ were considered statistically significant.

\section{Results}

\section{Clinicopathological Features of the Study Patients}

One hundred and seventy-four patients with cHCC-CCA were enrolled in this study, with an average age of $56.2 \pm 11.1$ years old, and $135(77.6 \%)$ patients were male. The mean interval time between MR imaging and surgery was $5.4 \pm 1.2$ days. There were 92 patients with CK-19 $\geq 5 \%$ and 82 patients with CK-19 $<5 \%$. The comparisons of clinicopathological features in cHCC-CCA patients stratified by CK-19 are detailed in Table 1. There were significant differences in HBV infection (77.2\% vs $89.0 \%, \mathrm{p}=0.04), \mathrm{CEA}>5 \mathrm{ng} / \mathrm{mL}(21.7 \%$ vs $9.8 \%$, $\mathrm{p}=0.03)$, and tumor size ( $\leq 2 \mathrm{~cm}: 15.2 \%$ vs $34.1 \% ; \geq 5 \mathrm{~cm}$ : $46.7 \%$ vs $24.4 \%, \mathrm{p}=0.002$ ) between the CK-19-positive and
CK-19-negative groups. However, age, sex, AFP $>20 \mathrm{ng} / \mathrm{mL}$, CA19-9 > $37 \mathrm{U} / \mathrm{mL}$, and MVI did not show significant differences between the CK-19-positive and CK-19-negative cHCC-CCA groups.

\section{MRI Characteristics}

The comparisons of MRI characteristics between CK-19positive and CK-19-negative patients are described in Table 2. The presence of some MR imaging features, such as mosaic architecture $(47.8 \%$ vs $32.9 \%, \mathrm{p}=0.046)$, targetoid appearance $(68.5 \%$ vs $52.4 \%, \mathrm{p}=0.03)$, cholangiectasis $(51.1 \%$ vs $31.7 \%, p=0.01)$, hepatic capsule retraction $(30.4 \%$ vs $14.6 \%, p=0.01)$, and corona enhancement $(50.0 \%$ vs $28.0 \%, p=0.003)$, was significantly higher in the CK-19-positive group than in the CK-19-negative group, while nonrim APHE was more common in the CK-19negative group $(51 / 82,62.2 \%)$ than in the CK-19-positive group $(43 / 92,46.7 \%)(p=0.04)$. In addition, no significant difference was found in other imaging features, including restricted diffusion, intratumoral hemorrhage, nonperipheral washout, enhancing capsule, nodule-in-nodule architecture, portal vein thrombus, and LI-RADS categorization (all $\mathrm{p}>$ 0.05), between the CK-19-positive group and the CK-19negative group (Figures 2 and 3 ).

\section{Uni-/Multivariate Analyses for Risk Factors for CK-19 in Patients with cHCC-CCA}

The univariate analysis showed that $\mathrm{HBV}$ infection $(\mathrm{OR}=$ $0.417, \mathrm{p}=0.04), \mathrm{CEA}>5 \mathrm{ng} / \mathrm{mL}(\mathrm{OR}=2.569, \mathrm{p}=0.04)$,

Table I Clinicopathological Characteristics of cHCC-CCA Patients

\begin{tabular}{|l|c|c|c|c|}
\hline \multirow{2}{*}{ Characteristics } & \multirow{2}{*}{ Total } & \multicolumn{2}{|c|}{ CK-19 } \\
\cline { 2 - 4 } & & Positive (n=92) & Negative (n=82) & P value \\
\hline Age (years) & & $56.6 \pm 11.2$ & $55.7 \pm 11.0$ & 0.57 \\
Sex (male) & $56.2 \pm 11.1$ & $74(80.4 \%)$ & $61(74.4 \%)$ & 0.34 \\
HBV infection & $135(77.6 \%)$ & $71(77.2 \%)$ & $73(89.0 \%)$ & 0.04 \\
AFP>20ng/mL & $144(82.8 \%)$ & $50(54.3 \%)$ & $46(56.1 \%)$ & 0.82 \\
CEA $>5 \mathrm{ng} / \mathrm{mL}$ & $96(55.2 \%)$ & $20(21.7 \%)$ & $8(9.8 \%)$ & 0.03 \\
CAI9-9>37U/mL & $28(16.1 \%)$ & $24(15.2 \%)$ & $18(22.0 \%)$ & 0.53 \\
MVI & $42(24.1 \%)$ & $41(44.6 \%)$ & $28(34.1 \%)$ & 0.16 \\
\hline Tumor size (cm) & $69(39.7 \%)$ & & & 0.002 \\
$\leq 2$ & & $14(15.2 \%)$ & $28(34.1 \%)$ & \\
$2-5$ & $42(24.1 \%)$ & $35(38.0 \%)$ & $34(41.5 \%)$ & \\
$\geq 5$ & $69(39.7 \%)$ & $43(46.7 \%)$ & $20(24.4 \%)$ & \\
\hline
\end{tabular}

Notes: *Data are mean \pm standard deviation. Except where labeled, data are numbers of patients, with percentages in parentheses.

Abbreviations: cHCC-CCA, combined hepatocellular carcinoma-cholangiocarcinoma; CK-19, cytokeratin 19; HBV, hepatitis B virus; AFP, alpha fetoprotein; CEA, carcinoembryonic antigen; CAI9-9, carbohydrate antigen 19-9; MVI, microvascular invasion. 
Table 2 Comparisons of Imaging Features Between the CK-19-Positive/Negative Groups

\begin{tabular}{|c|c|c|c|}
\hline Imaging Features & CK-19-Positive (n=92) & CK-19-Negative $(n=82)$ & p value \\
\hline Restricted diffusion & $86(93.5 \%)$ & 71 (86.6\%) & 0.13 \\
\hline Intratumoral hemorrhage & $21(22.8 \%)$ & II (I3.4\%) & 0.11 \\
\hline Non-rim APHE & $43(46.7 \%)$ & $5 I(62.2 \%)$ & 0.04 \\
\hline Non-peripheral washout & $58(63.0 \%)$ & 57 (69.5\%) & 0.37 \\
\hline Enhancing capsule & $53(57.6 \%)$ & $44(53.7 \%)$ & 0.60 \\
\hline Nodule-in-nodule architecture & $10(10.9 \%)$ & $8(9.8 \%)$ & 0.81 \\
\hline Mosaic architecture & $44(47.8 \%)$ & 27 (32.9\%) & 0.046 \\
\hline Targetoid appearance & $63(68.5 \%)$ & $43(52.4 \%)$ & 0.03 \\
\hline Cholangiectasis & 47 (51.1\%) & $26(31.7 \%)$ & 0.01 \\
\hline Hepatic capsule retraction & $28(30.4 \%)$ & $12(14.6 \%)$ & 0.01 \\
\hline Corona enhancement & $46(50.0 \%)$ & $23(28.0 \%)$ & 0.003 \\
\hline Portal vein thrombus & $19(20.7 \%)$ & $12(14.6 \%)$ & 0.30 \\
\hline LI-RADS categorization & & & 0.42 \\
\hline LR-3 & $5(5.4 \%)$ & $5(6.1 \%)$ & \\
\hline LR-4 & $2(2.2 \%)$ & $\mathrm{I}(\mathrm{I} .2 \%)$ & \\
\hline LR-5 & $20(21.7 \%)$ & $28(34.1 \%)$ & \\
\hline LR-M & $46(50.0 \%)$ & $36(43.9 \%)$ & \\
\hline LR-TIV & $19(20.7 \%)$ & $12(14.6 \%)$ & \\
\hline
\end{tabular}

Abbreviations: CK-19, cytokeratin 19; APHE, arterial phase hyperenhancement; LI-RADS, Liver Imaging Reporting and Data System.

tumor size $(\mathrm{OR}=2.075, \mathrm{p}=0.001)$, nonrim APHE $(\mathrm{OR}=$ 0.533, $\mathrm{p}=0.04)$, mosaic architecture $(\mathrm{OR}=1.867, \mathrm{p}=$ 0.047), targetoid appearance $(\mathrm{OR}=1.970, \mathrm{p}=0.03)$, cholangiectasis $(\mathrm{OR}=2.250, \mathrm{p}=0.01)$, hepatic capsule retraction $(\mathrm{OR}=2.552, \mathrm{p}=0.02)$, and corona enhancement $(\mathrm{OR}=2.565, \mathrm{p}=0.003)$ were significant risk factors for CK-19-positive cHCC-CCA. Unfortunately, the multivariate analysis revealed that only corona enhancement $(\mathrm{OR}=2.403, \mathrm{P}=0.03)$ was a significant independent risk factor associated with $\mathrm{CK}$-19-positive cHCC-CCA (Table 3). In addition, diagnostic performance of corona enhancement identifying CK-19-positive cHCC-CCA was also performed, including sensitivity (50\%), specificity (72\%), positive predictive value (PPV: 66.7\%), negative predictive value (NPV: $56.2 \%$ ), and area under curve (AUC: 0.574).

\section{Discussion}

Our study showed that the presence of corona enhancement was an independent predictor of $\mathrm{CK}-19$ positivity in patients with $\mathrm{cHCC}-\mathrm{CCA}$, indicating that $\mathrm{cHCC}-\mathrm{CCA}$ with CK-19 expression conforms to imaging features based on LI-RADS ver. 2018 but did not meet classic diagnostic criteria for HCC or ICC.

In the present study, we found that nonrim APHE was a differential factor between CK-19-positive and CK-19- negative cHCC-CCA only in the univariate analysis. Chung et $\mathrm{al}^{17}$ showed that $\mathrm{CK}-19$ was more frequently expressed in hypovascular HCC than in hypervascular tumors. Similarly, CK-19-negative cHCC-CCA showed stronger enhancement in the arterial phase than CK-19positive cHCC-CCA in our study. In addition, targetoid appearance, including target signs on DWI and rim APHE, peripheral washout, and delayed central enhancement on dynamic contrast-enhanced MR imaging, was also considered a valuable factor for predicting CK-19-positive cHCC-CCA. Previous studies suggested that rim APHE and/or target signs on DWI were significant predictors of small ICC due to the pathological features of ICC with peripheral hypercellularity and central stromal fibrosis. ${ }^{18,19}$ Fibrous stroma formation was more frequent in CK-19-positive HCC. ${ }^{7}$ Moreover, Park et $\mathrm{al}^{20}$ showed that rim APHE was correlated with poorer overall survival due to a lower amount of fibrotic stroma in cHCC-CCA. Delayed central enhancement might be an unfavorable predictor in ICC with regard to its fibrotic stroma. ${ }^{21}$ Asayama et $\mathrm{al}^{22}$ indicated that ICC patients with more than two-thirds delayed central enhancement had a poorer prognostic outcome than those with a small area.

Mosaic architecture is an ancillary feature of favoring HCC, which reflects the complex components of the tumor. Cholangiectasis and hepatic capsule retraction are 


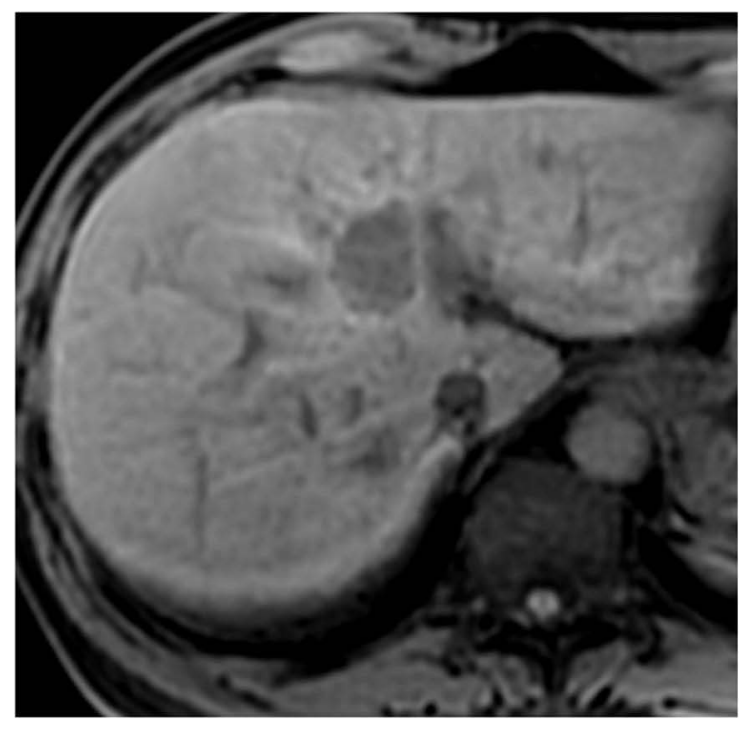

A

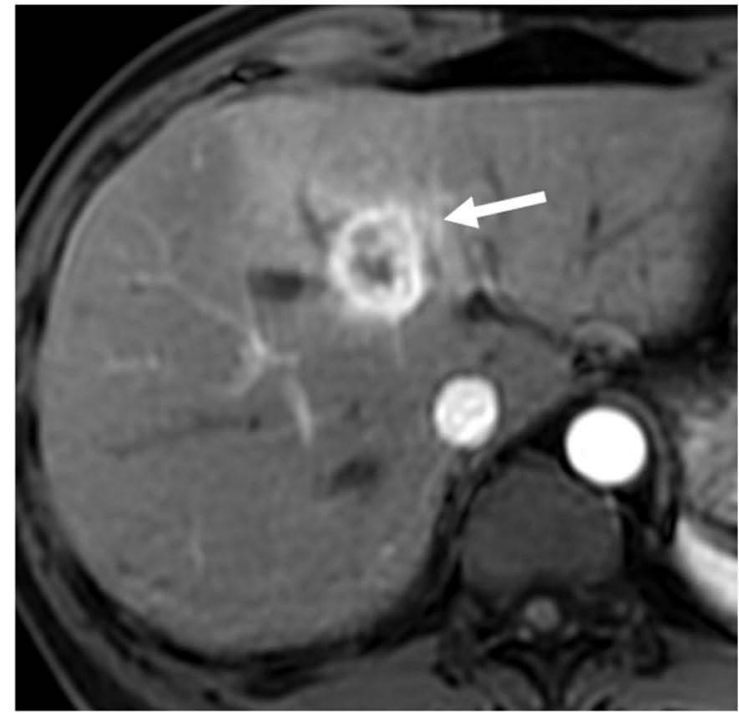

C

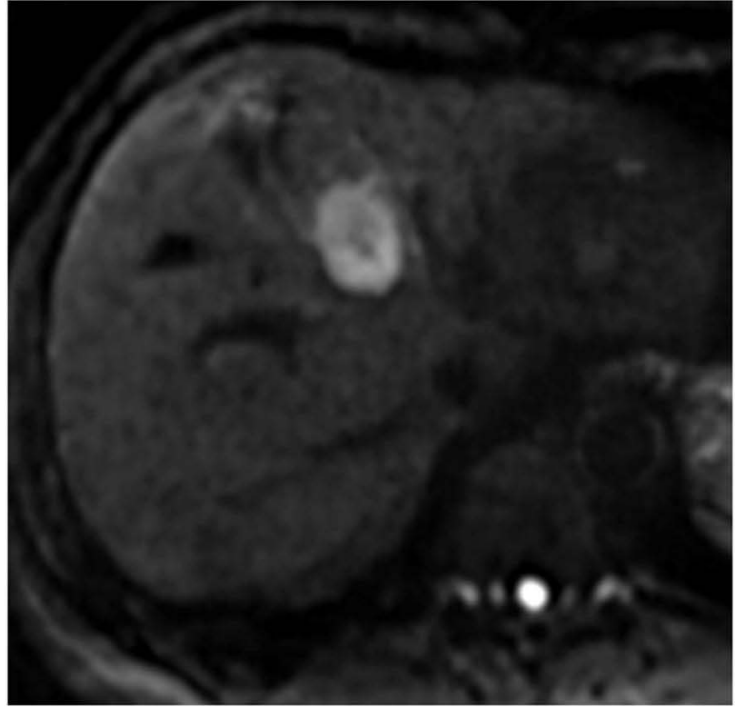

B

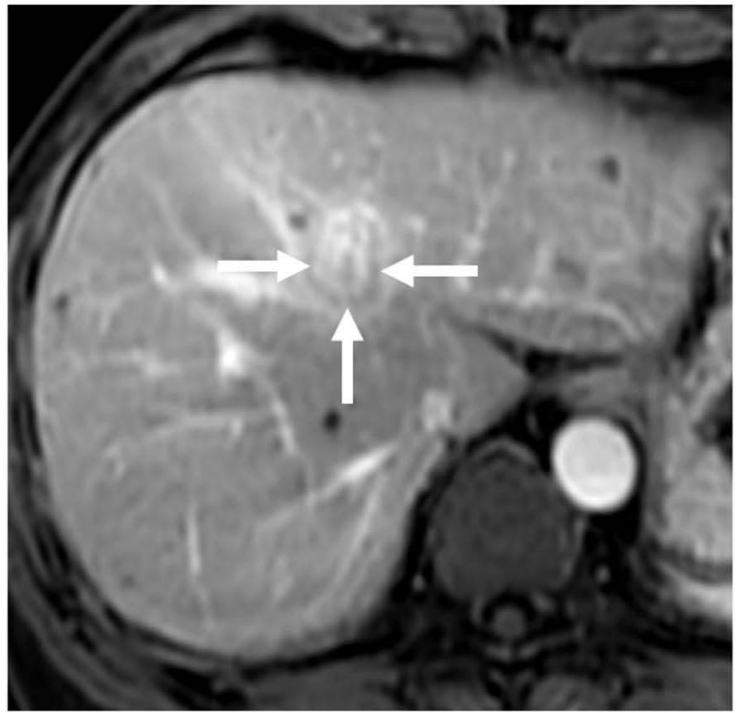

D

Figure 2 Images of a 56-year-old male with CK-19-positive cHCC-CCA. The lesion in left lobe of liver shows homogeneous hypointensity on TI-weighted imaging (A). There is a target sign with peripheral hyperintensity and central hypointensity on diffusion-weighted imaging (B). It presents rim arterial phase hyperenhancement and corona enhancement (arrow) on contrast-enhanced TI-weighted imaging (C) with contrast agent Gd-DTPA, and peripheral washout (arrows) and delayed central enhancement on portal venous phase (D).

ancillary features favoring ICC, indicating the invasion of malignant tumors. There are few findings about the correlation between these features and CK-19 expression in patients with HCC, ICC, and cHCC-CCA. However, in this study, mosaic architecture, cholangiectasis, and hepatic capsule retraction were important risk factors for CK19-positive cHCC-CCA in the univariate analysis, but they were not independent predictors. This may be explained by the fact that cHCC-CCA is a biphenotypic tumor with a heterogeneous mixture of both hepatocytic and cholangiocytic characteristics. ${ }^{23}$

In addition, corona enhancement is abnormal perfusion of liver tissue surrounding the tumor border in the late arterial phase or early portal venous phase, which represents compensatory hepatic arterial hyperperfusion surrounding the tumor due to portal branch microthrombosis. ${ }^{24} \mathrm{~A}$ previous study reported that irregular arterial peritumoral enhancement (OR: 0.322, $\mathrm{p}=$ 


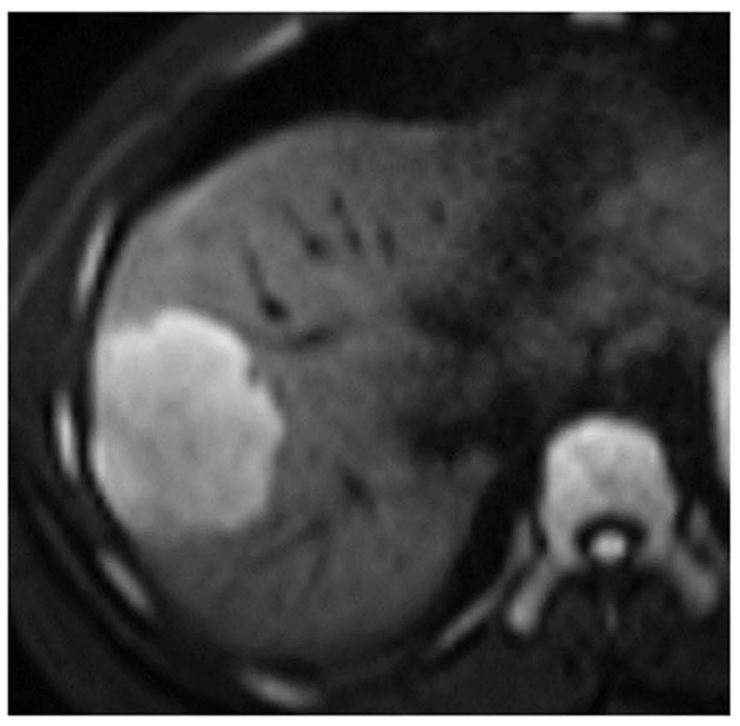

A

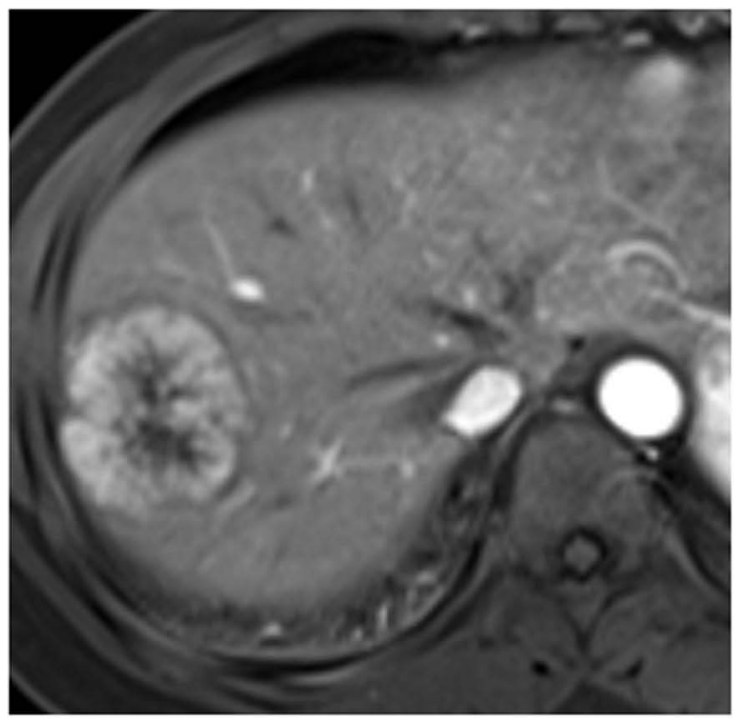

C

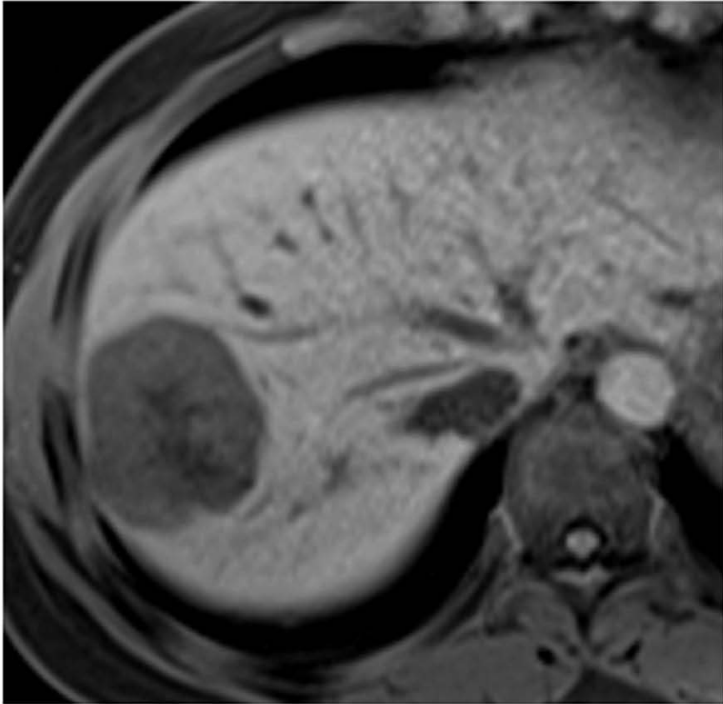

B

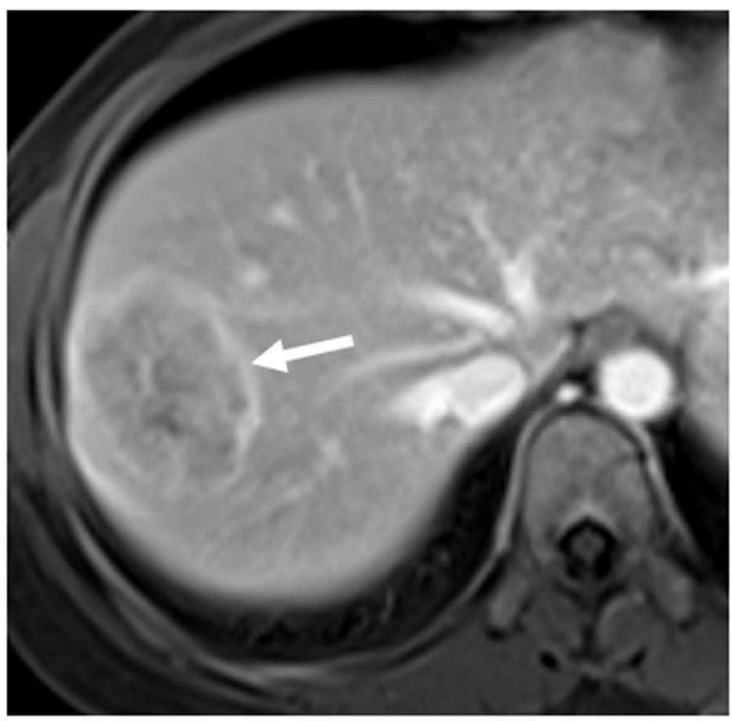

D

Figure 3 Images of a 32-year-old female with CK-19-negative cHCC-CCA. There is a round tumor in right lobe of liver showing targetoid restricted diffusion on diffusionweighted imaging (A). TI-weighted imaging shows heterogeneous hypointensity of the lesion (B). Nonrim arterial phase hyperenhancement (C), enhancing capsule (arrow) and delayed central enhancement are showed on dynamic contrast-enhanced TI-weighted imaging (D) with contrast agent Gd-DTPA.

0.001) was a predictor for MVI in cHCC-CCA. ${ }^{16}$ Therefore, our study showed that corona enhancement was a significant independent predictor of CK-19positive cHCC-CCA.

There are several limitations to this study. First, selection bias is inevitable, as this was a single-center and retrospective study. Therefore, we tried our best to select continuous patients and use multivariate analysis to make the results more objective. Second, the cHCC-CCA patients enrolled in this study were based on the updated
2019 WHO classification system, but they were not categorized in accordance with the classification system due to incomplete pathological data. Third, gadoxetic-acidenhanced MR imaging was not used in the present study, so we could not evaluate targetoid appearance on transitional phase and hepatobiliary phase described in the LIRADS ver. 2018. Finally, some of our results refer to HCC or ICC because of the few studies on cHCC-CCA; therefore, some larger multicenter prospective studies on cHCC-CCA are needed to validate our findings. 
Table 3 Uni/Multivariate Analyses for Risk Factors of CK-19 in Patients with cHCC-CCA

\begin{tabular}{|c|c|c|c|c|c|c|}
\hline \multirow[t]{2}{*}{ Characteristics } & \multicolumn{3}{|c|}{ Univariate Analysis for CK-19 } & \multicolumn{3}{|c|}{ Multivariate Analysis for CK-19 } \\
\hline & p value & OR & $95 \% \mathrm{Cl}$ & p value & OR & $95 \% \mathrm{Cl}$ \\
\hline Age (years) & 0.57 & 1.008 & $0.98 \mathrm{I}-1.036$ & & & \\
\hline Sex (male) & 0.34 & 0.707 & $0.346-1.444$ & & & \\
\hline HBV infection & 0.04 & 0.417 & $0.179-0.972$ & 0.14 & 0.472 & $0.173-1.289$ \\
\hline AFP $>20 \mathrm{ng} / \mathrm{mL}$ & 0.82 & 0.932 & $0.512-1.696$ & & & \\
\hline$C E A>5 n g / m L$ & 0.04 & 2.569 & $1.064-6.206$ & 0.09 & 2.479 & $0.877-7.133$ \\
\hline CA19-9>37U/mL & 0.53 & 1.255 & $0.623-2.527$ & & & \\
\hline MVI & 0.16 & 1.550 & $0.839-2.866$ & & & \\
\hline Tumor size $(\mathrm{cm})$ & 0.001 & 2.075 & $1.373-3.136$ & 0.15 & 1.577 & $0.853-2.917$ \\
\hline Restricted diffusion & 0.13 & 2.221 & $0.782-6.303$ & & & \\
\hline Intratumoral hemorrhage & 0.11 & 1.909 & $0.858-4.250$ & & & \\
\hline Non-rim APHE & 0.04 & 0.533 & $0.291-0.978$ & 0.61 & 1.333 & $0.448-3.986$ \\
\hline Non-peripheral washout & 0.37 & 0.748 & $0.397-1.409$ & & & \\
\hline Enhancing capsule & 0.60 & 1.174 & $0.644-2.137$ & & & \\
\hline Nodule-in-nodule architecture & 0.81 & 1.128 & $0.423-3.010$ & & & \\
\hline Mosaic architecture & 0.047 & 1.867 & $1.009-3.457$ & 0.94 & 1.032 & $0.433-2.455$ \\
\hline Targetoid appearance & 0.03 & 1.970 & $1.063-3.653$ & 0.49 & 1.427 & $0.516-3.948$ \\
\hline Cholangiectasis & 0.01 & 2.250 & $1.211-4.179$ & 0.11 & 1.937 & $0.864-4.341$ \\
\hline Hepatic capsule retraction & 0.02 & 2.552 & $1.198-5.438$ & 0.48 & 1.446 & $0.520-4.025$ \\
\hline Corona enhancement & 0.003 & 2.565 & $1.364-4.825$ & 0.03 & 2.403 & $1.093-5.283$ \\
\hline Portal vein thrombus & 0.30 & 1.518 & $0.687-3.357$ & & & \\
\hline
\end{tabular}

Abbreviations: cHCC-CCA, combined hepatocellular carcinoma-cholangiocarcinoma; CK-19, cytokeratin 19; OR, odds ratio; 95\% Cl, 95\% confidence interval; HBV, hepatitis B virus; AFP, alpha fetoprotein; CEA, carcinoembryonic antigen; CAI9-9, carbohydrate antigen 19-9; MVI, microvascular invasion; APHE, arterial phase hyperenhancement.

\section{Conclusion}

In conclusion, MR imaging featuring corona enhancement is significantly correlated with CK-19-positive patients with cHCC-CCA.

\section{Funding}

This study has received funding by National Natural Science Foundation of China (grant number 91859107), Shanghai Science and Technology Committee (grant number 18DZ1930102), Shanghai Science and Technology Committee (grant number 19411965500), Zhongshan Hospital, Fudan University (grant number 2018ZSLC22), Shanghai Municipal Key Clinical Specialty (grant number shslczdzk03202), Clinical Research Plan of SHDC (grant number SHDC2020CR1029B), and Zhongshan Hospital, Fudan University (grant number 2020ZSLC61).

\section{Disclosure}

All authors report no conflicts of interest in this work. All participants are informed about the purpose of the study, in accordance with the Declaration of Helsinki.

\section{References}

1. Chu KJ, Lu CD, Dong H, et al. Hepatitis B virus related combined hepatocellular-cholangiocarcinoma: clinicopathological and prognostic analysis of 390 cases. Eur J Gastroenterol Hepatol. 2014;26:192-199. doi:10.1097/MEG.0b013e3283625df9

2. Gera S, Ettel M, Acosta-Gonzalez G, et al. Clinical features, histology, and histogenesis of combined hepatocellular-cholangiocarcinoma. World J Hepatol. 2017;9:300-309. doi:10.4254/wjh.v9.i6.300

3. Jeon SK, Joo I, Lee DH, et al. Combined hepatocellular cholangiocarcinoma: LI-RADS v2017 categorisation for differential diagnosis and prognostication on gadoxetic acid-enhanced MR imaging. Eur Radiol. 2019;29:373-382. doi:10.1007/s00330-018-5605-x

4. Niu ZS, Niu XJ, Wang M. Management of hepatocellular carcinoma: predictive value of immunohistochemical markers for postoperative survival. World J Hepatol. 2015;7:49-69. doi:10.4254/wjh.v7.i1.7

5. Yuan RH, Jeng YM, Hu RH, et al. Role of $\mathrm{p} 53$ and beta-catenin mutations in conjunction with CK19 expression on early tumor recurrence and prognosis of hepatocellular carcinoma. $J$ Gastrointest Surg. 2011;15:321-329. doi:10.1007/s11605-010-1373-x

6. Chen J, Zhao Y, Lu XY, et al. Suppression of CK-19 expression by shRNA can inhibit the malignancy of hepatocellular carcinoma cells. Int $J$ Exp Med. 2018;11:3551-3559.

7. Kim H, Choi GH, Na DC, et al. Human hepatocellular carcinomas with "stemness"-related marker expression: keratin 19 expression and a poor prognosis. Hepatology. 2011;54:1707-1717. doi:10.1002/hep.24559

8. Govaere O, Komuta M, Berkers J, et al. Keratin 19: a key role player in the invasion of human hepatocellular carcinomas. Gut. 2014;63:674-685. doi:10.1136/gutjnl-2012-304351 
9. Lee JI, Lee JW, Kim JM, et al. Prognosis of hepatocellular carcinoma expressing cytokeratin 19: comparison with other liver cancers. World J Gastroenterol. 2012;18:4751-4757. doi:10.3748/wjg.v18. i34.4751

10. Chernyak V, Fowler KJ, Kamaya A, et al. Liver Imaging Reporting and Data System (LI-RADS) version 2018: imaging of hepatocellular carcinoma in at-risk patients. Radiology. 2018;289:816-830. doi:10.1148/radiol.2018181494

11. Lee HS, Kim MJ, An C. How to utilize LR-M features of the LI-RADS to improve the diagnosis of combined hepatocellular-cholangiocarcinoma on gadoxetate-enhanced MRI? Eur Radiol. 2019;29:2408-2416. doi:10.1007/s00330-018-5893-1

12. Choi SY, Kim SH, Park CK, et al. Imaging features of gadoxetic acid-enhanced and diffusion-weighted MR imaging for identifying cytokeratin 19-positive hepatocellular carcinoma: a retrospective observational study. Radiology. 2018;286:897-908. doi:10.1148/ radiol.2017162846

13. Hu XX, Wang WT, Yang L, et al. MR features based on LI-RADS identify cytokeratin 19 status of hepatocellular carcinomas. Eur J Radiol. 2019;113:7-14. doi:10.1016/j.ejrad.2019.01.036

14. Jeong HT, Kim MJ, Kim YE, et al. MRI features of hepatocellular carcinoma expressing progenitor cell markers. Liver Int. 2012;32:430-440.

15. Sempoux C, Kakar S, Kondo F, et al. Combined hepatocellularcholangiocarcinoma. In: Bosman FT, Carneiro F, Hruban RH, Theise ND, editors. WHO Classification of Tumours: Digestive System Tumours, 5th ed. Lyon: IARC; 2019:260-262.

16. Wang XL, Wang WT, Ma XJ, et al. Combined hepatocellular-cholangiocarcinoma: which preoperative clinical data and conventional MRI characteristics have value for the prediction of microvascular invasion and clinical significance? Eur Radiol. 2020;30:5337-5347. doi:10.1007/s00330-020-06861-2
17. Chung GE, Lee JH, Yoon JH, et al. Prognostic implications of tumor vascularity and its relationship to cytokeratin 19 expression in patients with hepatocellular carcinoma. Abdom Imaging. 2012;37:439-446. doi:10.1007/s00261-011-9756-3

18. Park HJ, Kim YK, Park MJ, et al. Small intrahepatic mass-forming cholangiocarcinoma: target sign on diffusion-weighted imaging for differentiation from hepatocellular carcinoma. Abdom Imaging. 2013;38:793-801. doi:10.1007/s00261-012-9943-x

19. Ni T, Shang XS, Wang WT, et al. Different MR features for differentiation of intrahepatic mass-forming cholangiocarcinoma from hepatocellular carcinoma according to tumor size. $\mathrm{Br} \mathrm{J}$ Radiol. 2018;5:20180017. doi:10.1259/bjr.20180017

20. Park SH, Lee SS, Yu E, et al. Combined hepatocellular-cholangiocarcinoma: gadoxetic acid-enhanced MRI findings correlated with pathologic features and prognosis. J Magn Reson Imaging. 2017;46:267-280. doi:10.1002/jmri.25568

21. Lacomis JM, Baron RL, Oliver JH, et al. Cholangiocarcinoma: delayed CT contrast enhancement patterns. Radiology. 1997;203:98-104. doi:10.1148/radiology.203.1.9122423

22. Asayama Y, Yoshimitsu K, Irie H, et al. Delayed-phase dynamic CT enhancement as a prognostic factor for mass-forming intrahepatic cholangiocarcinoma. Radiology. 2006;238:150-155. doi:10.1148/ radiol. 2381041765

23. Joo I, Kim H, Lee JM. Cancer stem cells in primary liver cancers: pathological concepts and imaging findings. Korean $J$ Radiol. 2015;16:50-68. doi:10.3348/kjr.2015.16.1.50

24. Choi JY, Lee JM, Sirlin CB. CT and MR imaging diagnosis and staging of hepatocellular carcinoma: part II. Extracellular agents, hepatobiliary agents, and ancillary imaging features. Radiology. 2014;273:30-50. doi:10.1148/radiol.14132362
Journal of Hepatocellular Carcinoma

\section{Publish your work in this journal}

The Journal of Hepatocellular Carcinoma is an international, peerreviewed, open access journal that offers a platform for the dissemination and study of clinical, translational and basic research findings in this rapidly developing field. Development in areas including, but not limited to, epidemiology, vaccination, hepatitis therapy, pathology and molecular tumor classification and prognostication are all considered for publication. The manuscript management system is completely online and includes a very quick and fair peer-review system, which is all easy to use. Visit http://www.dovepress.com/ testimonials.php to read real quotes from published authors. 